\title{
Archimedes Interdisciplinary Research Programme Forges a Broad Spectrum of Academic Innovations
}

\author{
Michalis Loupis, Georgios Fourlas, Petros Lampsas, Theodoros Tsiftsis, \\ Konstantinos Anagnostou, Nikos Strimpako, Yiannis Raftoyiannis, \\ Anastasia Pantera, Anna Deltsidou \\ TEl of Central Greece, Greece
}

\section{Abstract}

The basic goal of Archimedes III is to support research teams in Greek Technological Educational Institutes (TEI) and enhance their research capabilities by funding interdisciplinary and inter-institutional research. The programme aims also at attracting talented researchers and developing high quality research personnel. The TEls, prior to their advancement to the tertiary sector in 2000, focused only on educational activities. Since then, development of research became part of their mission. In order for the Ministry of Education to support TElsto develop their research capabilities, it designed research programmes targeting exclusively these organisations. Archimedes I and II were designed and ran in the previous programming period (2000-2006) while Archimedes III has been designed in the framework of the Operational Programme "Education and lifelong learning" of the current programming period, 2007-2013. The programme supports research projects in all research fields. However, particular emphasis is given to the fields of engineering and ICT where the main strengths of Greek Technological Educational Institutes are concentrated. The specific project of the TEl of Central Greece, described herewith, comprises 8 research subprojects in fields such as health, environment, energy and ICT with one additional subproject dedicated to management and dissemination. This paper is a comprehensive account of the research objectives and accomplishments of the research project as a whole.

Keywords: Academic Research Incentives, Energy, Environment, Health, ICT JEL classification: O31 Innovation and Invention: Processes and Incentives

Acknowledgments: Work supported under the Archimedes III Research Programme, NRSF 2007-2013

\section{Introduction}

The basic goal of Archimedes III is to support research teams in Greek Technological Educational Institutes (TEI) and enhance their research capabilities by funding interdisciplinary and inter-institutional research projects. The programme aims also at attracting talented researchers in TEls and developing high quality research personnel. TEls prior to their advancement to the tertiary sector in 2000 , focused only on educational activities. Since then, development of research became part of their mission. In order for the Ministry of Education to support TEls for developing their research capabilities, it designed research programmes targeting exclusively these organisations. Archimedes I and II were designed and ran in the previous programming period (2000-2006), while Archimedes III has been designed in the framework of the Operational Programme "Education and lifelong learning" of the 
current programming period 2007-2013. The programme supports research projects in all research fields. However, particular emphasis is given to the fields of engineering and ICT where the main strengths of Greek Technological Educational Institutes are concentrated. Moreover, $14 \%$ of the budget is allocated to outstanding proposals regardless of their research focus (Lyra, M., 2012). This paper attempts to describe the research initiatives undertaken within the specific project of the TEI of Central Greece. The project comprises 8 research subprojects in fields such as health, environment, energy and ICT with one additional subproject dedicated to management and dissemination. The structure of the paper follows that of the project and discusses briefly each of the 8 research projects, their current status and main results.

\section{Structural Analysis to Fault Diagnosis of a Mobile Robot}

In autonomous robotic systems, the occurrence of faults is unavoidable. This is mainly due to the fact that they consist of several subsystems that are subject to malfunction and also because the operating environment can appear unpredictable situations. A wheeled mobile robot is usually an embedded control platform which consists of an on-board computer, power, motor control system, communications, sonars, cameras, laser radar system and sensors such as gyroscope, encoders, accelerometer etc. Fault diagnosis and accommodation for such a system is a complicated process due to the numerous malfunctions that can be present such as faults of sensors and actuators. Previous research is focused only on the problem of fault detection and identification for wheeled robots and different approaches related to state estimation were introduced and on the problem of fault detection which is a separate problem in the fault diagnosis domain. Other research efforts are primarily intended to detect faults in the sensors of a wheeled robot. Concerning their search area of detection and accommodation on wheeled robots there is also a small number of efforts.

The aim of fault diagnosis is to produce the suitable fault statement regarding the malfunction of a wheeled robot. Among various approaches that allows generating residuals, only few deal with nonlinear systems. Structural analysis is a technique that provides feasible solutions to the residual generation of nonlinear systems. In this research, a model based fault diagnosis for a four-wheel skid steering mobile robot (SSMR) is proposed. The basic idea is to use structural analysis based technique in order to generate residuals. For this purpose we use the kinematic model of the mobile robot that serves to the design of the structural model of the system. This technique provides the parity equations which can be used as residual generators. The advantage of the proposed method is that offers feasible solution to the residual generation of nonlinear systems (Fourlas, G., 2014).

\section{A Centralized Architecture for Energy-Efficient Job Management in Data Centres}

Data Centres are facilities used to host cloud computing resources comprising computing systems and associated equipment, such as networking, storage, security and environmental control systems. An IDC, usually, deploys hundreds or thousands of blade servers, densely packed to maximize space utilization. With the rapid growth of IDCs in both quantity and scale over the last few years, the energy consumed by IDCs, directly related to the number of hosted servers and their workload, has skyrocketed. The most commonly used metric to determine the energy efficiency of a data centre is Power Usage Effectiveness (PUE). This simple 
ratio is the total power entering the data centre divided by the power used by the information technology equipment. However, only half of the large organizations (over 2000 servers) measure PUE in a detailed fashion, while only $18 \%$ of smaller data centres (those with fewer than 500 servers) had any PUE focus. This is an indication that there is a lot of space for optimizations, as far as IDC energy consumption is concerned.

Virtual Machine technology can be considered as a software alternative to the approaches that tackle energy efficiency in IDCs. VM technology enables multiple OS environments to co-exist on the same physical machine, albeit in strong isolation from each other. Despite their technical differences, all technologies support migration of virtual machines. There are two types of migration: regular migration and live migration. The former stops a VM, moves a VM from one host to another and then restarts the VM, while the latter transfers the $V M$ without ceasing to offer the service during transition. VMs may be transferred between physical machines, without user intervention, when certain conditions apply to the physical machine that originates the migration. VM and VM migration technologies exhibit great potential to efficiently manage workload consolidation, and therefore, improve the total IDC energy efficiency.

In this work, we implemented and tested Open Data Centre Manager (ODCM), a centralized mechanism that decides VM migrations (and consequently migrations of every job executed in this VM) according to a multi-criteria decision making algorithm and gathered monitoring information concerning computational load incurred in an IDC. VM migrations are decided in such a way that results in server consolidation, i.e. all the jobs submitted to a data centre run to as few servers as possible, taking into account Service Level Agreement between data centre managers and end users. We conducted an initial evaluation of ODCM in $a$, relatively small, cluster and initial results depict that ODCM may result in increased energy efficiency through server consolidation by employing live $\mathrm{VM}$ migrations (Perreas, G., Lampsas, P., 2014).

\section{Measurement and Analysis of Physicochemical Parameters Concerning in Natural Hot Spring Waters}

A source of considerable economic and scientific geological interest, underground hot water reservoirs, in the region of Thermopylae, can provide significant amounts of energy in profitable manner, via the development of suitable geothermal devices. Possible applications include the use of hot water for the heating of local buildings, spa, agricultural or industrial heating, etc. The aim of the present work is to develop a measurement system in order to investigate the physical and chemical characteristics of the Thermopylae natural hot water springs. This integrated system answers to the challenge for a system of continuous monitoring of physicochemical parameters in spring waters. The second target is to provide a thorough time series analysis of collected data.

The observed geophysical phenomena include variations in water level in deep wells, hydrostatic pressure, water flow rate from wells, electrical conductivity and water temperature. The most frequently studied geochemical phenomena have been concentrations of dissolved gas and ions in groundwater and variations in concentrations of crustal and mantle volatiles in ground gases. In the system under study four parameters were introduced and measured, which are important to geological deformations too: measuring water temperature to study the flow rate and depth variations, $\mathrm{pH}$ to study the acidity variations, redox potential to study the 
biologic load variations and electrical conductivity to study the salinity variations (Zarikas, V. et al, 2014).

\section{Design Considerations for Single-Phase Line Frequency Transformers Applied at Photovoltaic Systems}

Various improved inverter topologies have been recently presented for the connection of PV units to the grid. These topologies are replacing the classical full bridge one, making feasible the LFT elimination. This is an important advantage that leads to a drastic increase of the power density and the efficiency in PV applications. For example, $2.5 \mathrm{~kW}$ commercial single-phase inverters with LFT present roughly $92 \%$ European efficiency, while commercial solutions without LFT present over $96 \%$ and weigh $30 \%$ - 40\% less for the same power level. However, these improved topologies have to deal with the emerging leakage currents that produce electromagnetic compatibility and safety issues. As it concerns the elimination of leakage currents, additional semiconductor switches are incorporated, as well as high-frequency transformers (HFTs) and multiple output voltage levels (multilevel inverters). Last but not least, various sophisticated PWM patterns based on the space vector modulation have been proposed, proven to be very effective for the elimination of common mode voltages. These improved inverter topologies are characterized as transformerless if they do not include any LFT or HFT.

The above shows that the application of classical SPWM inverters with step-up LFT has been drastically reduced in grid-tied PV systems. Nevertheless, this topology remains very popular in standalone multisource installations where a diesel generator is combined with a PV generator, a battery bank, and/or a wind generator and especially when the application is a residential one. In more details, the presence of an LFT simplifies the construction of the necessary (for residential installations) earthed-neutral wire. Furthermore, the protection scheme of the installation (breaker sizing, implementation of protection selectivity, and so on) can be effectively designed by setting the transformer short circuit current (through its short circuit impedance setting).However, the presence of an LFT does not eliminate the generation of dc current components; the generation of a dc current component at the primary winding exhibits saturation issues, such as excessive heating of the transformer and the presence of even current and voltage harmonic components, due to the non-effective transformer design. Several technical solutions to this issue are being applied based on the appropriate transformer design. However, these solutions are not predictive, since they do not eventually prevent the generation of dc current components. On the other hand, several techniques have been proposed for the dc current blocking, focusing on the use of dc current sensors or series capacitors. Moreover, these solutions are increasing the inverter cost, while for some of them it is questionable whether they are effective under the existence of an LFT, since they have been proposed for transformerless inverter topologies (there is a possibility to excite resonances between the incorporate blocking capacitor and the LFT primary inductance). The main contribution of this work is the study of this phenomenon and the development of a technical solution, based on the optimal selection of the copper resistance at the primary winding, in order to achieve high power quality in the installations that are fed by classical single-phase SPWM inverters. It is noted that the proposed solution is additional to existing improved transformer design methods, aiming to the elimination of the dc current component (Papanikolaou, N. et al, 2015). 


\section{The 12-item oxford knee score: cross-cultural adaptation into Greek and assessment of its psychometric properties}

The Oxford knee score (OKS) is one of the most widely used scoring systems to assess function and pain for patients with total knee replacement (TKR) and its validation in different languages and cultures seems to be very important for obtaining reliable and valid data from these patients. The purpose of our work has been the crosscultural adaptation and assessment of psychometric properties of the OKS into Greek using translation protocols consistent with internationally recognised guidelines. For the cross-cultural adaptation, the back-translation procedure was utilised, comprising forward and backward translations by 4 bi-lingual translators. In the pilot study the questionnaire was completed from 20 subjects (10healthy and 10 patients with knee osteoarthritis) for linguistic validation and understanding. A further sample of42 patients with knee osteoarthritis (11 males, 31 females) aged 45-85 years old (Mean: 62.8, SD: 14.6) was included for the validity study. Subjects were requested to complete3 questionnaires; the OKS as developed in its final Greek version and two other questionnaires already adapted into Greek, the SF MC Gill Pain Questionnaire and the General Health Questionnaire (GHQ). Questionnaires were personally administered and completed via structured interviews by one physiotherapist. All interviews were taken place in a hospital orthopaedic clinic. A sample of 15 patients with TKR (2 males, 13 females) aged 66-77 years old (Mean: 70.53, SD: 3.8) was included for the reliability study. Subjects were requested to complete the OKS in two occasions, 7 days and one day before surgery. For the statistical analysis Pearson's correlation coefficient was used for the several correlations among questionnaires. Intra-class correlation coefficient(ICC 1,1$)$, Standard Error of Measurement (SEM), Smallest Detectable Difference (SDD), Cronbach $a$, and evaluation of floor and ceiling values in proportion of minimum and maximum scores were all used for the reliability study using the Statistical Package for the Social Sciences (SPSS, version 20.0).

Content validity of the Greek versions of the OKS questionnaire was achieved as all participants (including the ones in the pilot) found the questionnaires appropriate and comprehensible. Concurrent validity of OKS with SF MCGill Pain Questionnaire and $G H Q$ demonstrated a significant moderate to strong correlation $(r=0.75, p=$ $0.00 \& r=0.67, p=0.00$, respectively). Test-retest of OKS was excellent, with a very smaller $r$ or between measurements (ICC $=.93 ; \mathrm{Cl}=.82-.98 ; \mathrm{SEM}=0.99 ; \mathrm{SDD}=5.93 \%$ ). Internal consistency was also very high (Cronbach's $a=0.76$ ) and similar with relevant studies. No specific ceiling or floor effect was evident. The Greek version of the SSQOL questionnaire has proven to be valid, reliable, comprehensible and acceptable for the Greek knee patients tested (Strimpakos, N. et al, 2015).

\section{Regeneration of Abies cephalonica Loudon after a Large Fire in Central Greece}

Abies cephalonica Loudon (Greek fir) is a dominant tree species of the mountainous Greece that forms productive forests. Wildfires in fir forests were not considered a major threat but in recent decades, fir ecosystems in Greece have experienced large crown fires with subsequent ecological and economical losses. This study was designed to aid our understanding of Greek fir recovery after fire. In Central Greece, 12 years after a large fire, fir regeneration and site factors were studied in 143 sampling plots located in 10 transects. 
Fir regeneration density decreased abruptly with distance from the remnant stands and followed the negative exponential curve with decreasing regeneration abundance from the border of the unburned zone. Abiotic factors such as elevation, aspect, slope steepness and ground cover type could not significantly explain any variability in fir regeneration density. Our results showed that distance from a seed source was the most important variable in explaining fir regeneration. Moreover, the density of fir regeneration seemed adequate to provide stocking for a future fir forest.

\section{Floristic diversity of valonia oak silvopastoral woodlands in Greece}

Valonia oak (Quercus ithaburensis subsp. Macrolepis), a deciduous species grown in the xerothermic conditions of the east Mediterranean zone of Europe, forms woodlands of open canopy that permits adequate light to reach the ground thus sustaining an exceptionally rich flora. In Greece, these woodlands are mainly used for livestock grazing and considered important silvopastoral systems. Nowadays, most of them are abandoned and degraded as a result of inappropriate management. For the effective evaluation and sustainable management of these woodlands and the establishment of reliable monitoring systems, a detailed analysis of their plant diversity characteristics across Greece is needed.

This work aims at determining and comparing floristic diversity measures as well as at assessing the impact of human activities on vegetation and floristic diversity of the most characteristic valonia oak silvopastoral woodlands of Greece. It was found that the truncated log-normal model adequately predicts the species abundance data for eleven out of the twelve woodlands. From the statistical comparison of the diversity indices Q-statistic, Berger-Parker dominance index and Shannon-Wiener measure of equitability, three groups of these woodlands can be distinguished (a) the high-richness group of Pentalofos, Lesvos, Lakonia and Thesprotia, (b) the medium-richness group of Kriti Island, Alexandroupoli, Strofylia and Galaxidi, and (c) the low richness group of Kea Island, Amfilohia, Almyros and Lilaia. However, the Re'nyi's diversity ordering diagram showed that, generally, in low levels of entropy inconsistency is the rule and no clear pattern of diversity is extracted reflecting the impact of complex factors. Among them, those related to management seem to mostly determine plant diversity of valonia oak silvopastoral woodlands, with abiotic factors having an occasionally supporting role. Changes of species richness and evenness due to livestock grazing as well as acorn and wood harvesting are further discussed (Vrachnakis, M.S. et al, 2014)

\section{Non-invasive measurement method of hemodynamic parameters in students who smoke and consume caffeine}

The study involved a random sample of 178 students (19-35 years), including 94 women (52.8\%) and 84 men (47.2\%) who were divided into four groups: 1. Control group A (students who do not smoke and do not consume caffeine), 2. Group B, smokers (students who smoke but do not consume caffeine) 3. Group C, Caffeine (non-smokers who consume caffeine) and 4. Group D, smoking and caffeine (smoking students who consume caffeine). All participants completed the IPAQ-8 questionnaire, GHPSS, GLONIL, food consumption questionnaire, caffeinated beverages and demographics questionnaire and underwent oxygen saturation measurements, auto fluorescence (AgeReader) and hemodynamic parameters 
(Finometer) at rest and after consuming caffeine (group $C$ and D) and smoking (group B and D).

Values of AGEs (AgeReader) correlated significantly with age ( $r s=0.400, p$ $<0.001$ ), physical activity ( $r s=-0.242, p=0.002)$, smoking ( $r s=0.425, p<0.001)$, the years of smoking ( $r s=0.352, p=0.003$ ) and the number of cigarettes / day ( $r s=0.301$, $\mathrm{p}=0.009$ ). A statistically significant difference in the reported physical condition of smokers and non-smokers ( $t=3.715, p<0.001$ ) was observed as well as a statistically significantly higher rate of AGEs in smoking students ( $t=-5.943, p<0.001$ ). The statistically significant difference in the price of AGEs found between smokers and non-smokers, renders the group of smokers more vulnerable to cardiovascular diseases. In the smokers' subgroup $31.9 \%$ showed mild behavioural dependence, $56.9 \%$ moderate, $8.4 \%$ strong and $2.8 \%$ very strong behavioural dependence on smoking habits. Regarding the blood pressure it was found that a) in B Group both systolic and diastolic blood pressure rose during smoking (tSAP $=-7.941, p<0.001$ and tDAP $=-9.147, p<0.001$ ) b) similarly, in Group $C$ both systolic and diastolic blood pressure rose 1 hour after caffeine consumption (tSAP $=-4.724, p<0.001$ and tDAP $=-$ 3.676, $\mathrm{p}=0.001) \mathrm{c}$ ) in Group $\mathrm{D}$ both systolic and diastolic blood pressure rose during smoking (tSAP $=-7.077, \mathrm{p}<0.001$ and tDAP $=-6.810, \mathrm{p}<0.001$ ) and one hour after consumption of caffeine (tSAP $=-6.148, p<0.001$ and tDAP $=-5.545, p<0.001$ ) (Deltsidou, A., 2015)

\section{Conclusion}

Integration of research and education is of utmost importance for any educational system and has been the driving force of fundamental research in Greece. The best ideas often come from the bottom up, i.e. from researchers themselves and some of the most innovative ideas come from young researchers who are newly tenured or untenured. Important research breakthroughs have been indeed effectuated by people who, against substantial cultural if not economic odds, have reached out to other fields, merging different perspectives and creating new ideas, even new fields. The ARCHIMEDES III Program is such an example, already producing significant research results across a multiplicity of scientific domains. The research projects described in this paper will be concluded by the November 2015 and although fairly limited in funding, each of them will have produce significant results in the context of the research interests of our academic staff.

\section{References}

1. Deltsidou, A. (2015), "Non-invasive measurement method of hemodynamic parameters and of the level of final products of non-enzymatic glycosylation in students who smoke and consume caffeine", Working paper, TEl of Central Greece, Lamia, May 2015.

2. Fourlas, G.K. (2014), "On the Application of Structural Analysis to Fault Diagnosis of aMobile Robot", IEEE International Conference onRobotics and Biomimetics.

3. Lyra, M. (2012), " Archimedes III ", available at: http://erawatch.jrc.ec.europa.eu/erawatch/opencms/information/country_pages/gr/su pportmeasure/support_mig_0002/accessed (accessed April 17th 2015)

4. Papanikolaou, N., Kyritsis, A., Loupis, M., Tzotzos, Ch., Zoga, E. (2015) "Design considerations for single phase line frequency transformers applied at photovoltaic systems", IEEE Power and Energy Technology Systems Journal, Vol. 2, pp. 1-12.

5. Perreas, G., Lampsas, P. (2014), "A Centralized Architecture for Energy-Efficient Job Management in Data Centers", CLOUD COMPUTING 2014: The Fifth International Conference on Cloud Computing, GRIDs, and Virtualization. 
6. Raftoyiannis, Y., Spanos, I. (2015), "Regeneration of Abies cephalonica Loudon after a Large Fire in Central Greece", Southeast Europe, Vol. 6 No 1, pp. 5-14.

7. Strimpakos, N., Dapka, Papachristou A., F., Kapreli, E. (2015), "The 12-item oxford knee score: cross-cultural adaptation into Greek and assessment of its psychometric properties", Physiotherapy, Vol. 101, No. 1, pp. 1445 -1446.

8. Vrahnakis, M.S., Fotiadis, G., Pantera, A., Papadopoulos, A., Papanastasis, V.P. (2014), "Floristic diversity of valonia oak silvopastoral woodlands in Greece", Agroforestry Systems, Vol. 88 No 5, pp. 877-893.

9. Zarikas, V., Anagnostou, K.E., Avlakiotis, P., Kotsopoulos, S., Liolios, C., Latsos, T., Perantzakis, G., Lygdis, A., Lykourgiotis, A., Antoniou, D. (2014), "Measurement and Analysis of Physicochemical Parameters Concerning Thermopylae Natural Hot Spring Waters", Journal of Applied Sciences, Vol. 14 No 19, pp. 2331-2340.

\section{About the authors}

Michael I. LOUPIS is an Assistant Professor at the Department of Electrical Engineering of the Technological Educational Institute (T.E.I.) of Central Greece, Greece. Author can be contacted at mloupis@teiste.gr

George. K. FOURLAS is an Assistant Professor at the Department of Informatics of the TEl of Central Greece, Greece. Author can be contacted at gfourlas@teiste.gr

Petros LAMPSAS is a Professor at the Department of Informatics of the TEl of Central Greece, Greece. Author can be contacted at plam@teiste.gr

Theodoros A. TSIFTSIS is an Associate Professor at the Department of Electrical Engineering of the TEl of Central Greece, Greece. Author can be contacted at tsiftsis@teiste.gr

Konstantinos ANAGNOSTOU is a Professor at the Department of Electrical Engineering of the TEl of Central Greece, Greece. Author can be contacted at anagko@teiste.gr

Nikolaos STRIMPAKOS is a Professor at the Department of Physiotherapy of the TEl of Central Greece, Greece. Author can be contacted at nikstrimp@teiste.gr

Yiannis RAFTOYIANNIS is a Professor at the Department of Forestry of the TEl of Central Greece, Greece. Author can be contacted at rafto@teiste.gr

Anastasia PANTERA is a Professor at the Department of Forestry of the TEl of Central Greece, Greece. Author can be contacted at pantera@teiste.gr

Anna DELTSIDOU is an Associate Professor at the Department of Midwifery of the TEI of Athens, Greece. Author can be contacted at deltsidou@teiste.gr 